\title{
A Rare Side Effect of Intravesical Bacillus Calmette-Guérin Therapy: Reactive Arthritis
}

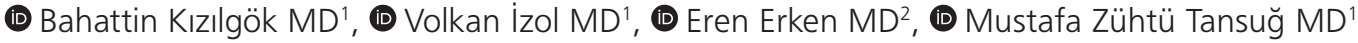 \\ 'Çukurova University Faculty of Medicine, Department of Urology, Adana, Turkey \\ ${ }^{2}$ Çukurova University Faculty of Medicine, Department of Internal Diseases, Division of Rheumatology, Adana, Turkey
}

\begin{abstract}
Approximately $70-80 \%$ of bladder cancers are superficial tumors and not muscle invasive. Complete transurethral resection of the bladder tumour (TUR-BT) is the standard approach to these patients. Intravesical treatments such as adriamycin, doxorubicin, epirubicin, mitomycin-c and Bacillus Calmette-Guérin (BCG) may be performed after TUR-BT in order to prevent further recurrence or progression. BCG is generally used in high-risk patients and causes local or systemic side effects in less than $5 \%$ of patients. Osteoarticular side effects are very rare and usually manifest as joint pain and arthritis (\%0.5-1). In this case report, we present the management of reactive arthritis in a patient treated with intravesical BCG for bladder cancer. Keywords: Intravesical Bacillus Calmette-Guérin, bladder cancer, reactive arthritis
\end{abstract}

\section{Introduction}

Bladder cancer is the seventh most common cancer in males worldwide, and eleventh most common when both genders are considered. It is 3-4 times more common in men than in women, especially after 60 years of age. Histopathologically, more than $90 \%$ of bladder cancers are transitional cell carcinomas and $70-80 \%$ are muscle non-invasive. Approximately $70 \%$ of noninvasive bladder cancers are Ta tumors, 20\% are T1 tumors, and $10 \%$ are carcinoma in situ (1). The treatment approach in these cases is complete transurethral resection of bladder tumor (TUR-BT). A well-performed TUR-BT allows correct staging and administration of intravesical treatment, which can prevent recurrence or progression $(2,3,4)$. These intravesical treatments include adriamycin, doxorubicin, epirubicin, mitomycin-c, and Bacillus Calmette-Guérin (BCG).

BCG is obtained from an attenuated strain of Mycobacterium bovis (5). Intravesical BCG application, performed since 1976, is believed to have an antitumor effect $(5,6,7)$. Severe local or systemic side effects related to BCG occur in less than $5 \%$ of patients. Local side effects include cystitis findings, hematuria, granulomatous prostatitis, and epididymuritis. Systemic side effects may manifest as fever, allergic reaction, sepsis, arthralgia, or arthritis $(8,9)$. Of these adverse systemic effects, osteoarticular involvement is rare, occurring in $1-5 \%$ of cases. Osteoarticular side effects usually manifest clinically with joint pain and arthritis $(0.5-1 \%)(10,11)$. In this case report, we present a patient who developed reactive arthritis after undergoing TUR-BT for nonmuscle-invasive bladder cancer and intravesical BCG treatment.

\section{Case Report}

Ultrasound examination in a 43-year-old male patient with hematuria revealed a pelvic mass approximately $3 \mathrm{~cm}$ in diameter. In cystoscopy, a papillary formation about $3 \mathrm{~cm}$ in size was observed on the left sidewall and was resected. Following TUR-BT, T1G3 was identified in histopathological examination and intravesicular BCG therapy was planned. The first instillation was done on postoperative day 18. After the fifth instillation, the patient developed complaints of fever $\left(38.2{ }^{\circ} \mathrm{C}\right)$, fatigue, and pain, redness, and tenderness in his right big toe. The following day he developed pain in the right knee and shoulder joints and was readmitted to the hospital. After consultation with the rheumatology department, laboratory analysis of complete blood count, acute phase reactants, complete urinalysis, urine culture, and serological markers was requested. Complete blood

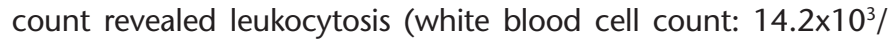
$\mu \mathrm{L}$ ), acute phase reactants were elevated (C-reactive protein: $15.7 \mathrm{mg} / \mathrm{dL}$ ), and erythrocyte sedimentation rate was $18 \mathrm{~mm} / \mathrm{L}$. Urinalysis and urine culture were normal. Serologic examination was negative for rheumatoid factor negative and positive for human leukocyte antigen (HLA)-B27. Anteroposterior X-rays of the right foot and knee were taken. X-ray to investigate the cause of swelling in the knee revealed no evidence other than edema in the soft tissue (Figure 1). The orthopedics and traumatology

Address for Correspondence: Bahattin Kızılgök MD, Çukurova University Faculty of Medicine, Department of Urology, Adana, Turkey E-mail: bahattinkizilgok@gmail.com ORCID-ID: orcid.org/0000-0002-7732-1545 Received: 15.09.2017 Accepted: 04.10.2017 
department was consulted and magnetic resonance imaging (MRI) of the right knee joint was requested. MRI of the right knee joint revealed fluid accumulation around the medial and collateral ligaments. Samples of the fluid were obtained under sterile conditions. On microscopic examination, 10-12 leukocytes were observed in each field. Cultures of the fluid were negative. Therefore, a diagnosis of septic arthritis was excluded.

Reactive arthritis secondary to intravesical BCG treatment was suspected due to fever, oligoarthritis, and HLA-B27 positivity. The patient was treated with prednisolone $(32 \mathrm{mg} /$ day for 7 days, followed by $24 \mathrm{mg} /$ day), betamethasone (once a week), and diclofenac sodium. Steroid therapy was tapered and discontinued after 3 months, and the patient's complaints resolved completely (Figure 2 ).

The patient provided informed consent for his case to be presented in this report.

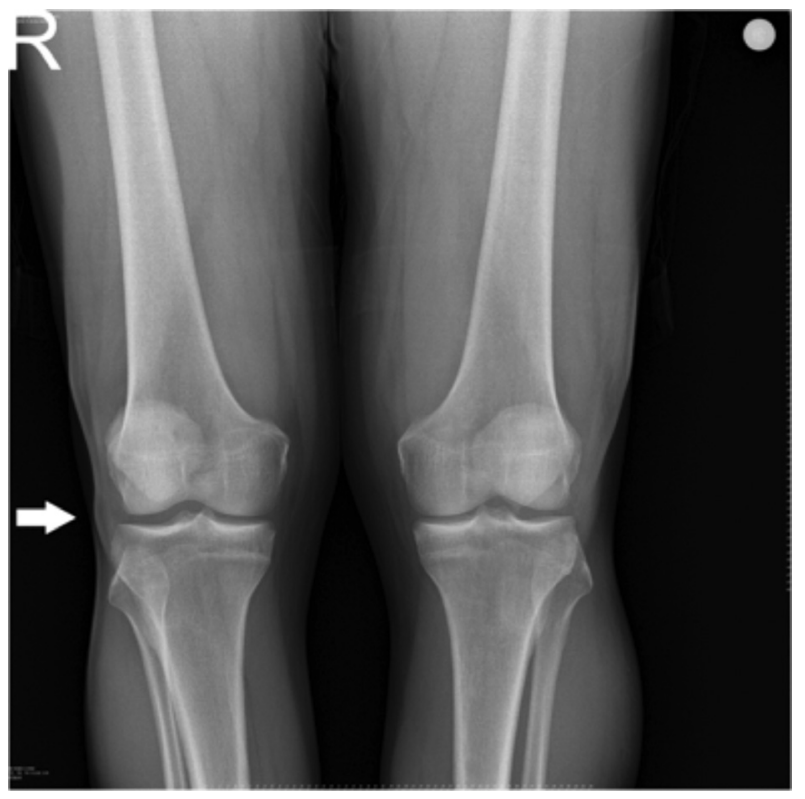

Figure 1. Soft tissue edema in the patient's right knee

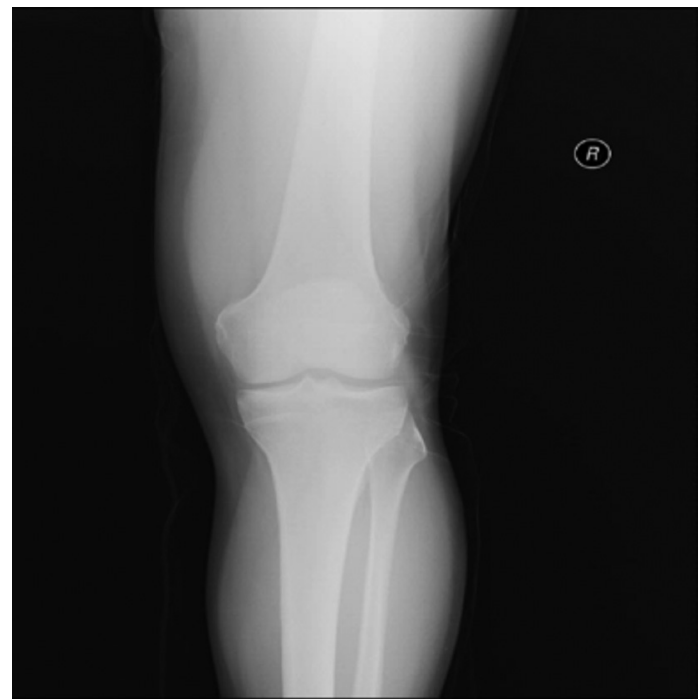

Figure 2. The knee appears normal after treatment

\section{Discussion}

Osteoarticular side effects rarely develop due to intravesical BCG therapy. In a 2006 review, Tinazzi et al. (6) screened 48 articles and classified 61 autoimmune complications developed after intravesical BCG treatment. They reported that $64 \%$ of the patients developed joint pain and arthritis, 24\% had Reiter's syndrome, $4 \%$ had arthritis and fever, $2 \%$ had psoriatic arthritis, and $2 \%$ had Sjogren's syndrome.

Reactive arthritis secondary to intravesical BCG treatment is usually seen in the fifth and sixth decades. It typically occurs after the fourth or fifth instillation. Onset occurred after the fifth instillation in our case. It is characterized by asymmetric oligoarticular involvement. The most commonly involved joints are the knee, wrist, and ankle. In addition to arthritis, patients may exhibit dactylitis, urethritis, and uveitis $(7,12,13,14)$. Elevated acute phase reactants, inflammatory response in the synovial fluid, and negative mycobacterial cultures of synovial fluid are expected findings of laboratory studies. Other findings should raise suspicion of septic arthritis. Blood and urine analyses negative for infection support a diagnosis of reactive arthritis $(7,9,11)$. We suspected reactive arthritis in our case due to the asymmetric articular involvement as well as the patient's high acute phase reactants levels and our findings of 10-12 leukocytes/field and negative cultures of fluid obtained from the knee.

The mechanism by which reactive arthritis develops secondary to intravesical BCG therapy is not yet clear. In a case report published in 2002, Pardalidis et al. (15) claimed that mycobacterial heat shock protein 65 shared similar homology to human cartilage tissue, and suggested that this may cause cross-reaction and increase cytokine production. The increase in cytokine release activates CD8+ T cells, and cellular immunity may lead to tissue damage (15). This autoimmune response is more common after intravesical BCG treatment in individuals who are positive for HLA-B27 and B7 (6). About $60 \%$ of patients are HLA-B27 positive, as was our patient.

Reactive arthritis secondary to intravesical BCG therapy resolves with treatment in most cases. The condition may become chronic in small proportion of patients, but they can be treated with non-steroidal anti-inflammatory drugs (NSAIDs) and corticosteroids either alone or in combination, and if necessary, immunosuppressive treatments such as methotrexate can be used $(6,7)$.

Antituberculous therapy may also be needed in some patients who do not respond to treatment (7). The condition resolved in our patient after a successful three months of treatment with combined corticosteroid and NSAID.

Reactive arthritis is a rare side effect of intravesical BCG therapy. Despite the low incidence of osteoarticular side effects, it should be remembered that such reactions are more likely to occur in HLA-B27 and HLA-B7 positive patients.

In the event of osteoarticular side effects, the patient should be evaluated in collaboration with relevant departments, particularly rheumatology, to determine whether the clinical presentation is inflammatory in nature, to rule out septic arthritis, and to initiate treatment as early as possible. 


\section{Ethics}

Informed Consent: It was taken.

Peer-review: Internally peer-reviewed.

\section{Authorship Contributions}

Surgical and Medical Practices: V.I., Concept: B.K., V.İ., Design: B.K., Data Collection or Processing: B.K., Analysis or Interpretation: M.Z.T., E.E., Literature Search: B.K., Writing: B.K. Conflict of Interest: No conflict of interest was declared by the authors.

Financial Disclosure: The authors declared that this study received no financial support.

\section{References}

1. Torre LA, Bray F, Siegal RL, et al. Global Cancer Statistics, 2012. Ca Cancer J Clın 2015;65:87-108.

2. Cheng L, Neumann RM, Weaver AL, et al. Predicting cancer progression in patients with stage T1 bladder carcinoma. J Clin Oncol 1999;17:3182-3187.

3. Peyromaure M, Zerbib M. T1G3 transitional cell carcinoma of the bladder: recurrence, progression and survival. BJU Int 2004;93:60-63.

4. Iori F, Di Seri M, De Nunzio C, et al. Long-term maintenance bacille Calmette-Guérin therapy in high-grade superficial bladder cancer. Urology 2002;59:414-418.

5. Morales A, Eidinger D, Bruce AW. Intracavitary Bacillus CalmetteGuerin in the treatment of superficial bladder tumors. J Urol 1976;116:180-183.
6. Tinazzi E, Ficarra V, Simeoni S, et al. Reactive arthritis follow-ing BCC immunotherapy for urinary bladder carcinoma: a systematic review. Rheumatol Int 2006;26:481-488.

7. Macía Villa C, Sifuentes Giraldo W, Boteanu A, et al. Reactive Arthritis After the Intravesical Instillation of BCG. Reumatol Clin 2012;8:284-286.

8. Babjuk M, Burger M, Compérat E, et al. EAU Guidelines on Nonmuscle-invasive Bladder Cancer (TaT1 and CIS). European Association of Urology 2018.

9. Clavel G, Grados F, Lefauveau P, Fardellone P. Osteoarticular side effects of BCG therapy. Joint Bone Spine 2006;73:24-28. Epub 2005 Mar 24.

10. Orihuela E, Herr HW, Pinsky CM, Whitmore Jr WF. Toxicity of intravesical BCG and its management in patients with superficial bladder tumors. Cancer 1987;60:326-333.

11. Henares P, Barbadillo C, Cruz M. Artritis reactiva secundaria a inmunoterapia intravesical con bacilo. Semergen 2006;32:176-178.

12. Manzini $C U$, Bernini $L$, Elkhaldi $N$, et al. actilitis and oligoarthritis after BCG immunotherapy in a patient affected by bladder cancer. Reumatismo 2006;58:230-232.

13. Chevrel G, Zech C, Miossec P. Severe uveitis followed by reactive arthritis afterbacillus Calmette-Guérin therapy. J Rheumatol 1999;26:1011.

14. Belmatoug $N$, Levy-Djebbour S, Appelboom T, et al. Polyarthritis in 4 patients treated with intravesical BCG-therapy for carcinoma of the bladder. Rev Rhum Ed Fr 1993;60:162-166.

15. Pardalidis NP, Papatsoris AG, Kosmaoglou EV, Georganas C. Two cases of acute polyarthritis secondary to intravesicalBCGadjuvant therapy for superficial bladder cancer. Clin Rheumatol 2002;21:536-537. 